\title{
The nonlinear optical rectification and second harmonic generation in asymmetrical Gaussian potential quantum well: Effects of hydrostatic pressure, temperature and magnetic field
}

\author{
Xin Liu ${ }^{1}$, LiLi Zou ${ }^{1}$, Chenglin Liu ${ }^{1}$, Zhi-Hai Zhang ${ }^{1 *}$, Jian-Hui Yuan ${ }^{2 * *}$ \\ ${ }^{1}$ School of New Energy and Electronic Engineering, Yancheng Teachers \\ University, Yancheng, 224051, China \\ ${ }^{2}$ Department of Physics, Guangxi medical university, Nanning, Guangxi, 530021, China
}

\begin{abstract}
In the present work, the effects of hydrostatic pressure, temperature, and magnetic field on the nonlinear optical rectification (OR) and second-harmonic generation (SHG) in asymmetrical Gaussian potential quantum well (QW) have been investigated theoretically. Here, the expressions for the optical properties are calculated by the compact-density-matrix approach and iterative method. Simultaneously, the energy eigenvalues and their corresponding eigenfunctions have been obtained by using the finite difference method. The energy eigenvalues and the shape of the confined potential are modulated by the hydrostatic pressure, temperature, and magnetic field. So the results of a number of numerical experiments indicate that the nonlinear OR and SHG strongly depends on the hydrostatic pressure, temperature, and magnetic field. This gives a new degree of freedom in various device applications based on the intersubband transitions of electrons.
\end{abstract}

Keywords: Optical rectification; Second-harmonic generation; Hydrostatic pressure; Temperature; Magnetic field.

\footnotetext{
${ }^{*}$ Corresponding author

${ }^{* *}$ Corresponding author

Email addresses: zhangzhihai3344@mail.bnu.edu.cn; Tel: 183-6114-8893 (Zhi-Hai Zhang ${ }^{1}$ ), jianhui831110@163.com; Tel:150-7882-3937 (Jian-Hui Yuan²)
} 


\section{Introduction}

The low-dimensional systems have huge nonlinearities (nonlinear OR, SHG, Third-harmonic generation, absorption coefficients, and refractive index chages). Such properties have become important physical foundations for many optoelectronic devices, for example semiconductor lasers[1], solar cells[2], quantum computing[3], optical memories [4] and infrared photodetectors[5]. With recent rapid advances of molecular beam epitaxy and metal-organic chemical vapour deposition $[6,7]$, it has become possible to produce a variety of dimensionality semiconductor nanostructures $[8,9,10]$, which have enabled electrons to be confined in semiconductor nanostructures. Especially, much attention has been paid to the larger-band-gap low-dimensional semiconductor QWs structure because they are more advantageous to grow, process, and fabricate into devices than are small-band-gap semiconductors.

Among nonlinear optical properties related to intersubband transitions in QWs which were composed by different materials, OR and SHG have attracted much attention. We know that the second-order nonlinear susceptibility is negligible except for a small contribution from the bulk susceptibility in a symmetric QW structure, but as the symmetry can be broken by applying an electric field to a symmetric QW, nonvanishing contributions to the second-order nonlinear optical susceptibilities are expected to appear. For instance, K.X. Guo et al. published in 1993 have studied the applied electric field on the nonlinear OR in GaAs/AlGaAs parabolic QWs. They found the OR is six orders of magnitude higher than in bulk GaAs using a compact density-matrix approach[11]. In 2002, J.M. Jancu et al. investigated AlGaSb L-valley QWs. They found the AlGaSb L-valley QWs system is suitable for detection throughout the infrared spectrum, which strongly enhance absorption properties for long wavelength infrared detection by considering asymmetric structures[12]. In 2012, J. Zhu et al. investigated the intersubband absorption in strained $\mathrm{Al}_{x} \mathrm{Ga}_{1-x} \mathrm{~N} / \mathrm{GaN}$ QWs with $\mathrm{In}_{y} \mathrm{Ga}_{1-y} \mathrm{~N}$ nanogroove layers. Their results indicate that the position and height of absorption peaks are sensitive to the structural parameters such as In composition, nanogroove thickness and the strain induced by the groove layer[13]. The intense laser field effects on the linear and nonlinear intersubband optical properties in a strained InGaN/GaN QW have been studied by M.J. Karimi and H. Nafaei[14]. They found the resonant peaks experience a redshift(blue-shift) with the increase in the laser field intensity and well width(In composition). 
Recently, there is a considerable interest in the optical phenomena based on different materials semiconductor QWs nanostructure in the presence of some external perturbations, such as electric field, magnetic field, hydrostatic pressure and temperature. In particular, the effects of the hydrostatic pressure and temperature on optical properties of nanostructures have also been studied by several authors. The work by Lepkowski and Majewski on the pressure dependence of the elastic constants of zinc-blende InN and GaN, and its consequence on the light emission in cubic InGaN/GaN QWs[15]. The hydrogenic impurity states in InGaN/GaN quantum well wires with applied pressure are the subject of the paper by Baser et al.[16]. And the properties of donor impurity states in multiple zinc-blende InGaN/GaN QWs with applied hydrostatic pressure are investigated in Ref.[17]. I Karabulut et al. published in 2011, have investigated the combined effect of applied electric and magnetic fields and hydrostatic pressure on the nonlinear optical rectification and optical absorption in GaAs $/ \mathrm{Ga}_{1-x} \mathrm{Al}_{x}$ As asymmetric double QW. The results show that the combination of hydrostatic pressure and electric fields is a tool that allows the coupling of both QWs and provide a way of tuning the position of the maxima and minima of the nonlinear optical properties[18]. C.M. Duque et al. analyzed the effect of the hydrostatic pressure on the optical properties in zinc-blende GaN/InGaN QWs. They have found that the influence of the hydrostatic pressure implies the increase of the exciton binding energy, effect that is less pronounced for higher values of the In concentration[19]. Majority of provided results indicate that the various factors such as temperature, applied electric and magnetic field, and hydrostatic pressure affect the electronic and optical properties of these semiconductor materials. Particularly, the manipulation of hydrostatic pressure and temperature leads to change in the confinement of electrons and in different transitions, which is very useful to fabricated optoelectronics devices.

The paper is organized as follows: In the section 2, without any approximation, the eigenfunctions and eigenenergies of electron states of asymmetrical Gaussian potential QW are obtained using finite difference method, and the analytical expression for the OR coefficient and SHG susceptibility are derived by means of the compact-density-matrix approach and an iterative method. In the section 3, the numerical results and discussions are presented for asymmetrical Gaussian potential QW under the simultaneous effects of hydrostatic pressure, temperature, and applied magnetic field. A brief summary is given in section 4 . 


\section{Theory}

As shown in Fig.1, we consider the problem of an electron confined in the asymmetrical Gaussian potential QW grown along the z-axis, under the influence of applied magnetic field (magnetic field perpendicularly to the growth direction), the hydrostatic pressure as well as temperature. The Hamiltonian of the system can be written as[20]

$$
\left(-\frac{\hbar}{2} \frac{d}{d_{z}}\left[\frac{1}{m^{*}(P, T)} \frac{d}{d_{z}}\right]+V(z, P, T)+\frac{e^{2} B^{2}}{2 m^{*}(P, T) c^{2}}\right) \varphi(z)=E \varphi(z),
$$

where $z$ represents the growth direction of the QWs. $B$ is the external magnetic field, and $T$ is the temperature. $m^{*}(P, T)$ is the pressure- and temperature-dependent electron effective mass in the well region, given by the expression[21, 22]

$$
\frac{m_{0}}{m^{*}(P, T)}=1+E_{p}^{\Gamma}\left[\frac{2}{E_{g}^{\Gamma}(P, T)}+\frac{1}{E_{g}^{\Gamma}(P, T)+\Delta_{0}}\right],
$$

where $m_{0}$ is the free electron mass, $E_{p}^{\Gamma}=7.51 \mathrm{eV}$ is the energy related to the momentum matrix element, $\Delta_{0}=0.341 \mathrm{eV}$ is the spin-orbit splitting, and $E_{g}^{\Gamma}(P, T)$ is the variation of the energy gap for a GaAs semiconductor at the $\Gamma$-point with the temperature and hydrostatic pressure, given by[21]

$$
E_{g}^{\Gamma}(P, T)=E_{g}^{\Gamma}(0, T)+b P+c P^{2},
$$

where $E_{g}^{\Gamma}(0, T)=\left[1.519-\left(5.405 \times 10^{-4} T^{2}\right) /(T+204)\right] \mathrm{eV}, b=1.26 \times 10^{-2}$ $\mathrm{eV} / \mathrm{kbar}$ and $c=-3.77 \times 10^{-5} \mathrm{eV} / \mathrm{kbar}^{2}$.

And the confinement potential $V(z, P, T)$ is given by

$$
V(z, P, T)=\left\{\begin{array}{lc}
-V_{0}(P, T) \exp \left(-z^{2} / 2 L(P)^{2}\right) & z \geq 0 \\
\infty & z<0,
\end{array}\right.
$$

here the barrier height is given by[23]

$$
V_{0}(p, T)=Q_{c} \Delta E_{g}^{\Gamma}(X, P, T)
$$

where $Q_{c}=0.6$ is the conduction band offset parameter, $X=0.3$ is the mole fraction of aluminum in $G a_{1-x} A l_{x} A s$, and $\Delta E_{g}^{\Gamma}(X, P, T)$ is the band 
gap difference between QW and barrier matrix at the $\Gamma$-point as a funtion of $P$ and $T$, which is given by[24]

$$
\Delta E_{g}^{\Gamma}(X, P, T)=\Delta E_{g}^{\Gamma}(X)+P D(X)+G(X) T,
$$

where $\Delta E_{g}^{\Gamma}(X)=\left(1.55 X+0.37 X^{2}\right) \mathrm{eV}$ is the variation of the gap difference and $D(X)=\left[-\left(1.3 \times 10^{-3}\right) X\right] \mathrm{eV} / \mathrm{kbar}, G(X)=\left[-\left(1.11 \times 10^{-4}\right) X\right] \mathrm{eV} / \mathrm{K}$. The pressure-dependent width of the QWs is given by

$$
L(P)=L(0)\left(1-\left(S_{11}+2 S_{12}\right) P\right) .
$$

where $S_{11}=1.16 \times 10^{-3} \mathrm{kbar}^{-1}$ and $S_{12}=3.7 \times 10^{-4} \mathrm{kbar}^{-1}$ are the elastic constants of the GaAs and $L(0)$ is the original width of the confinement potential in $\mathrm{z}=$ direction.

To obtain energy eigenvalues and eigenfunctions under the influence of the applied magnetic fields, hydrostatic pressure and temperature in asymmetrical Gaussian potential QW. Based on discretization of the Hamiltonian on a spatial grid, the Schrödinger equation (Eq.(1)) under finite difference method can be written as follows:

$$
\begin{aligned}
& -\frac{\hbar}{2 m^{*}(P, T)}\left[\frac{\varphi\left(z_{j+1}\right)-2 \varphi\left(z_{j}\right)+\varphi\left(z_{j-1}\right)}{h^{2}}\right]+V\left(z_{j}, P, T\right) \varphi\left(z_{j}\right) \\
& +\frac{e^{2} B^{2}}{2 m^{*}(P, T) c^{2}} \varphi\left(z_{j}\right)=E \varphi\left(z_{j}\right) .
\end{aligned}
$$

where $h=z_{j+1}-z_{j}$ is the spacing between the two neighboring discrete points. The matrix (Eq.(8)) is diagonalized to obtain the eigenvalues and the wavefunctions of the asymmetrical Gaussian potential QW. After the energies and their corresponding wave functions are obtained, the OR coefficients and SHG susceptibilities can be calculated in asymmetrical Gaussian potential QW by the compact-density-matrix method and the iterative procedure[25, $26,27]$. The system is excited by electromagnetic field $\vec{E}(t)=\tilde{E} e^{i \omega t}+\tilde{E} e^{-i \omega t}$. Let us denote $\rho$ as the one-electron density matrix for this regime. Then the evolution of density matrix $\rho$ obeys the following:

$$
\frac{\partial \rho_{i j}}{\partial t}=\frac{1}{\hbar}\left[H_{0}-q z \vec{F}(t), \rho\right]_{i j}-\Gamma_{i j}\left(\rho-\rho^{(0)}\right)_{i j} .
$$

where $H_{0}$ is the Hamiltonian for this system without the electromagnetic field $\vec{E}(t), \rho^{(0)}$ is the density matrix and $\Gamma_{i j}$ is the relaxation rate. 
Eq.(9) is calculated by the following iterative method:

$$
\rho(t)=\sum_{n} \rho^{(n)}(t)
$$

with

$$
\frac{\partial \rho_{i j}^{(n+1)}}{\partial t}=\frac{1}{i \hbar}\left\{\left[H_{0}, \rho^{(n+1)}\right]_{i j}-i \hbar \Gamma_{i j} \rho_{i j}^{(n+1)}\right\}-\frac{1}{i \hbar}\left[q z, \rho^{(0)}\right]_{i j} \vec{E}(t) .
$$

The electric polarization of the QWs due to $\vec{E}(t)$ can be expressed as

$$
p(t)=\left(\varepsilon_{0} \chi^{(1)} \tilde{E} e^{i \omega t}+\varepsilon_{0} \chi_{2 \omega}^{(2)} \tilde{E}^{2} e^{2 i \omega t}\right)+\text { c.c. }+\varepsilon_{0} \chi_{0}^{(2)} \tilde{E}^{2},
$$

where $\chi^{(1)}, \chi_{0}^{(2)}$ and $\chi_{2 \omega}^{(2)}$ are the linear susceptibility, OR and second-harmonic generation, respectively. $\varepsilon_{0}$ is the vacuum dielectric constant. The electronic polarization of the $n t h$ order is given as

$$
p^{(n)}=\frac{1}{V} \operatorname{Tr}\left(\rho^{(n)} e z\right)
$$

where $V$ is the volume of interaction and Tr denotes the trace or summation over the diagonal elements of the matrix $\rho^{(n)} e z$. In our paper, the OR coefficients per unit volume is given as

$$
\begin{gathered}
\chi_{0}^{(2)}=\frac{4 e^{3} \sigma_{\nu}}{\varepsilon_{0} \hbar^{2}} \mu_{01} \delta_{01} \frac{\omega_{01}^{2}\left(1+\frac{\Gamma_{2}}{\Gamma_{1}}\right)+\left(\omega^{2}+\frac{1}{T_{2}^{2}}\right)\left(\frac{\Gamma_{2}}{\Gamma_{1}}-1\right)}{\left[\left(\omega_{01}-\omega\right)^{2}+\Gamma_{2}^{2}\right]\left[\left(\omega_{01}+\omega\right)^{2}+\Gamma_{2}^{2}\right]}, \\
\chi_{2 \omega}^{(2)}=\frac{e^{3} \sigma_{\nu}}{\varepsilon_{0}} \frac{\mu_{01} \mu_{12} \mu_{20}}{\left(\hbar \omega-E_{10}-i \hbar \Gamma_{0}\right)\left(2 \hbar \omega-E_{20}-i \hbar \Gamma_{0}\right)} .
\end{gathered}
$$

where $\sigma_{\nu}$ is the density of electrons in the QWs, $\mu_{i j}=\left|\left\langle\Psi_{i}|z| \Psi_{j}\right\rangle\right|(i, j=$ $0,1,2)$ is the off-diagonal matrix element, $\delta_{01}=\left|\mu_{11}-\mu_{00}\right|, \omega_{01}$ is the transition frequency, and $\Gamma_{k}=1 / T_{k}$ with $k=(0,1,2)$ are damping terms associated with the lifetime of the electrons involved in the transitions.

\section{Results and discussions}

In this section, we will discuss the OR coefficient and the SHG susceptibility in asymmetrical Gaussian potential QW under the influence of 
combined independent external magnetic fields, temperature as well as hydrostatic pressure. The parameter adopted in our calculation are as follows: $\varepsilon_{0}=8.85 \times 10^{-12} \mathrm{Fm}^{-1}, T_{1}=1 \mathrm{ps}, T_{2}=0.2 \mathrm{ps}, T_{0}=0.14 \mathrm{ps}$, and $\sigma_{\nu}=5 \times 10^{24} \mathrm{~m}^{-3}$.

In Fig.1(a)-(c) we show the confinement potential and the schematic representation of the density of probability for the single-electron wavefunctions of the lowest two confinement states in asymmetrical Gaussian potential QW for different values of hydrostatic pressure (a), temperature (b) and applied magnetic fields (c), respectively. We observe that the well depth of the QW decreases with the increasing hydrostatic pressure and temperature, so that the subband energy levels are mostly localized in the upper part of well. But the well width has a very small variation with the hydrostatic pressure and temperature. The effective well width of the QW can be significantly reduced with the enhancement of applied magnetic field. Also the ground and first excited energy levels become farther to each other since the magnetic field applied perpendicular to the growth direction brings an additional geometric confinement on the electronic state in asymmetrical Gaussian potential QW.

In Fig.2, the OR coefficient $\chi_{0}^{(2)}$ is plotted as a function of the incident photon energy for three different values of $P($ a), the first two energy levels, the energy difference $E_{21}$ between ground states $E_{1}$ and first excited states $E_{2}$ (b) are depicted versus the hydrostatic pressure $P$ with $B=0 \mathrm{~T}, T=0 \mathrm{~K}$, $L=8 \mathrm{~nm}$. From Fig.2(a), the following points can be deduced: (1) The magnitude of the OR coefficient decreases as the hydrostatic pressure increases. The physical reason is that the hydrostatic pressure has an effect similar to reducing the confinement, which increases the electrons effective mass, and this in turn results in a decrease of the strength confinement[28]. To look at this behavior accurately, the dipole matrix element $\mu_{01} \delta_{01}$ is plotted as a function of the hydrostatic pressure. As the hydrostatic pressure increases, the dipole matrix element decreases(see the inset in Fig.2(a)). (2) The OR coefficient has a maximum value when photon energy equal to the energy difference between ground state and the first excited state, which is the resonance photon energy. (3) The resonant peaks of OR coefficient move to the lower energy region as the hydrostatic pressure increases. The physical origin of this behavior is the variations of the energy difference between ground state and first excited state by varying the hydrostatic pressure. Fig.2(b) reveals that the energy difference between ground state and first excited state decreases with the hydrostatic pressure increases. Therefore, by increasing the hydrostatic pressure the resonant peaks shift to lower energy regions, 
which is good agreement with Fig.2(a).

In Fig.3, the OR coefficient $\chi_{0}^{(2)}$ is plotted as a function of the incident photon energy for three different values of $T(\mathrm{a})$, the first two energy levels, the energy difference $E_{21}$ between ground states $E_{1}$ and first excited states $E_{2}(\mathrm{~b})$ are depicted versus the temperature $T$ with $B=0 \mathrm{~T}, P=0 \mathrm{kbar}$, $L=8 \mathrm{~nm}$. From Fig.3(a), it can be clearly seen that the resonant peaks of OR coefficient are directly related to the temperature and increase by increasing the temperature, which is attributed to the enhanced dipole matrix element $\mu_{01} \delta_{01}$ (see the inset in Fig.3(a)). And the resonant peaks experience a slight red-shift with the increase in the temperature. It is due to the decrease in energy difference between ground state and first excited state, which is good agreement with Fig.3(b). Fig.3(b) reveals that the ground state increases slightly with the increasing of the temperature. However, the first excited state hardly changed. So the energy difference between the two different electronic states decreases slightly when the temperature $T$ increases.

The OR coefficient $\chi_{0}^{(2)}$ as a function of the incident photon energy with $P=0 \mathrm{kbar}, T=0 \mathrm{~K}, L=8 \mathrm{~nm}$ is shown in Fig.4(a). Several distinct values of the applied magnetic field: $B=0 \mathrm{~T}, B=15 \mathrm{~T}$ and $B=30 \mathrm{~T}$ are taken into account. We noted that the OR coefficient has strongly related to the applied magnetic field. Increasing applied magnetic field weakens and blueshifts the resonant peaks of OR coefficient. These features can be understood easily as follows: the well width reduces with the enhancement of the applied magnetic field (see Fig.1(c)), which leads to the stronger quantum confinement, so the energy difference between the first excited state and the ground state of the system increases, and also the corresponding peak position shifts toward higher energies (see Fig.4(b)). In addition, to show the relation between the OR coefficient and applied magnetic field more clearly, we plot the variation of the dipole matrix element $\mu_{01} \delta_{01}$ as a function of the applied magnetic field. It is observed that the dipole matrix element significantly decreases with applied magnetic field strengths.

Fig.5(a) displays the SHG susceptibility $\chi_{2 \omega}^{(2)}$ versus the photon energy for three different values of hydrostatic pressure $P$ with $B=0 \mathrm{~T}, T=0 \mathrm{~K}, L=8$ $\mathrm{nm}$. It is easily seen that (1) the resonant peaks of the SHG susceptibility decreases and the blue-shift of resonance of photon is induced as the strength of hydrostatic pressure increases. (2) the two peaks of the SHG susceptibility corresponding to the two single-photon resonances, the main and the weak peaks are always related to the resonant energy $E \approx E_{20} / 2$ and $E \approx E_{10}$. 
In order to interpret these properties above, the energy difference $E_{20}$ and $E_{10}$ are depicted as a function of the hydrostatic pressure. We find that the energy difference $E_{20}$ and $E_{10}$ decrease with the increase of the hydrostatic pressure (see Fig.5(b)). Thus we can easily understand the cause of the blue-shift of resonance of photon.

In Fig.6, the SHG susceptibility $\chi_{2 \omega}^{(2)}$ is plotted as a function of the incident photon energy for three different values of temperature $T(\mathrm{a})$, the energy difference $E_{10}$ and $E_{20}(\mathrm{~b})$ are depicted versus the temperature $T$ with $B=0$ T, $P=0$ kbar, $L=8 \mathrm{~nm}$. These figure show that the resonant peaks of the SHG susceptibility experience a red-shift with the increase. This feature can be explained by Fig.6(b), which presents the energy difference decreases with the enhancement of the temperature, which will lead to a red shift of resonant peak. It is also seen that the peak values are related to the values of temperature. The magnitude of resonant peaks decrease with increasing temperature. These results can be explained as follows: the resonant peaks occur at $E \approx E_{20} / 2$ and $E \approx E_{10}$ and the SHG susceptibility is maximum when $E \approx E_{20} / 2$. Simultaneously, the distance $\left(E_{20} / 2-E_{10}\right)$ between the positions of the main and weak peaks increase with increasing temperature. According to Eq.(15), the SHG susceptibility is proportional to $\mu_{01} \mu_{12} \mu_{20} /\left(E_{20} / 2-E_{10}\right)$, variation of $\left(E_{20} / 2-E_{10}\right)$ term is dominant and hence, the SHG susceptibility decreases with increasing temperature.

In Fig.7, the SHG susceptibility $\chi_{2 \omega}^{(2)}$ is plotted as a function of the incident photon energy for three different values of applied magnetic field $B(\mathrm{a})$, the energy difference $E_{10}$ and $E_{20}(\mathrm{~b})$ are depicted versus the applied magnetic field $B$ with $T=0 \mathrm{~K}, P=0$ kbar, $L=8 \mathrm{~nm}$. From Fig.7(a), it is easily found that a transition from two single-photon resonances to a two-photon resonance will appear adjusted by the applied magnetic field, where the single peak of the SHG susceptibility corresponding to the two-photon resonance about the photon energy $E \approx E_{20} \approx E_{10}$. It is also seen that the resonant peaks of the SHG susceptibility move toward the high energy regions(blue shift) as the applied magnetic field increases. This is because that the energy difference increases as the applied magnetic field increases(see Fig.7(b)).

\section{Conclusions}

In this paper, we have calculated the OR coefficients and SHG susceptibility for asymmetrical Gaussian potential QW by using the compact density matrix approach. The related energy eigenvalues and the energy eigenfunc- 
tions have been obtained by the finite difference method. Our results can be summarized as follows: (i)the hydrostatic pressure and temperature redshift the resonant peaks of the OR coefficients and SHG susceptibility, whereas the applied magnetic field shift these peaks into the red end. (ii)the magnitude of resonant peaks of OR coefficients decrease with increasing hydrostatic pressure and temperature. However the magnitude of resonant peaks of OR coefficients is increasing with increasing of the applied magnetic field.(iii)the SHG susceptibility can be adjusted by the hydrostatic pressure, temperature and the applied magnetic field, which a transition from a two single-photon resonances to two-photon resonance will appear. (iv)the order of the magnitude of the nonlinear OR is $10^{3}$ times large than the magnitude of the SHG susceptibility when taking the same parameters. Our theoretical results may make a contribution to experimental studies and provide a kind of approximative modeling for the practical application such as optoelectronics devices and optical communication.

Acknowledgments: Project supported by the National Science Foundation of China (under Grant No. 11447193, 11447101, 61178003 and 61475039), the Youth Science Foundation of Guangxi Medical University in China (under Grant No. GXMUYSF201313), the Guangxi Department of Education Research Projects in China (under Grant No. KY2015LX046) and the University National Science Foundation of Jiangsu Province of China (under Grant No. 14KJB140015).

\section{References}

[1] N. Kristaedter, O.G. Schmidt, N.N. Ledentsov, D. Bimberg, V.M. Ustinov, A.Yu. Egorov, A.E. Zhukov, M.V. Maximov, P.S. Kopev, Zh.I. Alferov, Appl. Phys. Lett. 69 (1996) 1226.

[2] E. Leobandung, L.J. Guo, S.Y. Chou, Appl. Phys. Lett. 67 (1995) 2338.

[3] F. Durante, P. Alves, G. Karunasiri, N. Hanson, M. Byloos, H.C. Liu, A. Bezinger, M. Buchanan, Infrared Phys. Technol. 50 (2007) 182.

[4] K. Imamura, Y. Sugiyama, Y. Nakata, N. Muto, N. Yokoyama, Jpn. J. Appl. Phys. 34 (1995) L1445.

[5] R.F. Kazarinov, R.A. Suris, Sov. Phys. Semicond. 5 (1971) 707. 
[6] P.D. Dapkus, Annu, Rev. Mater. Sci. 12 (1982) 243.

[7] S. Hersee, J.P. Duchemim, Annu, Rev. Mater. Sci. 12 (1982) 65.

[8] M. Sundaram, S.A. Chalmers, P.E. Hopkins, A.C. Gossard, Science 254 (1991) 1326.

[9] M.R. Panish, Science 208 (1980) 916.

[10] C. Sirtori, F. Capasso, D.L. Sivco, A.Y. Cho, Phys. Rev. Lett. 68 (1992) 1010.

[11] K.X. Guo, S.W. Gu, Phys. Rev. B 47 (1993) 16322.

[12] J.M. Jancu, F. Bassani, P. Voisin, J. Appl. Phys. 92 (2002) 641-643.

[13] J. Zhu, S.L. Ban, S.H. Ha, Superlatt. and Microstruct. 51 (2012) 471479 .

[14] M.J. Karimi, H. Vafaei, Physica B 452 (2014) 131-135.

[15] S.P. Lepkowski, J.A. Majewski, Solid State Commun. 131 (2004) 763.

[16] P. Baser, S. Elagoz, N. Baraz, Physica E 44 (2011) 356.

[17] T. Wang, Z. Zeng, C. Xia, S. Wei, Superlatt. and Microstruct. 49 (2014) 211.

[18] İ. Karabulut, M.E. Mora-Ramos, C.A. Duque, J. Lumin. 131 (2011) 1502-1509.

[19] C.M. Duque, A.L. Morales, M.E. Mora-Ramos, C.A. Duque, Phys. Status Solidi B 4 (2015) 670-677.

[20] N. Eseanu, E.C. Niculescu, L.M. Burileanu, Physica E 41 (2009) 1386.

[21] H. Ehrenrich, J. Appl. Phys. 32 (1961) 2155.

[22] D.E. Aspnes, Phys. Rev. B 14 (1976) 5331.

[23] R.F. Kopf, M.H. Herman, M.L. Schnoes, A.P. Perley, G. Livescu, M. Ohring, J. Appl. Phys. 71 (1992) 5004.

[24] A. Montes, A.L. Morales, C.A. Duque, Surf. Rev. Lett. 9 (2002) 1753. 
[25] J.H. Yuan, N. Chen, Y. Zhang, H. Mo, Z.H. Zhang, Physica E 77 (2016) 102-107.

[26] H. Hassanabadi, G.H. Liu, L.L. Lu, Solid State Commun. 152 (2012) 1761-1766.

[27] M.J. Karimi, H. Vafaei, Superlatt. and Microstruct. 78 (2015) 1-11.

[28] L. Bouzaiene, R. Ben Mahrsia, M. Baira, L. Sfaxi, H. Maaref, J. Lumin. 135 (2013) 271. 


\section{Figure Captions}

Figure 1 The variation of the confinement potential profile the first two states and squared envelope wave functions related to these first two states are depicted as a function of the position $\mathrm{z}$ for different values of (a)the hydrostatic pressure $P=0,50,100$ kbar with $T=0 \mathrm{~K}, B=0 \mathrm{~T}, L=8 \mathrm{~nm}$, (b)temperature $T=0,200,400 \mathrm{~K}$ with $P=0 \mathrm{kbar}, B=0 \mathrm{~T}, L=8 \mathrm{~nm}$, and (c)applied magnetic field $B=0,15,30 \mathrm{~T}$ with $P=0 \mathrm{kbar}, T=0 \mathrm{~K}, L=8$ nm.

Figure 2 The OR coefficient $\chi_{0}^{(2)}$ versus the incident photon energy with three different strength of the hydrostatic pressure $P(\mathrm{a})$, and the low-lying energy levels of $E_{0}, E_{1}$, the difference $E_{10}(\mathrm{~b})$ versus the hydrostatic pressure for $B=0 \mathrm{~T}, T=0 \mathrm{~K}, L=8 \mathrm{~nm}$.

Figure 3 The OR coefficient $\chi_{0}^{(2)}$ versus the incident photon energy with three different strength of the temperature $T(\mathrm{a})$, and the low-lying energy levels of $E_{0}, E_{1}$, the difference $E_{10}(\mathrm{~b})$ versus the temperature for $B=0 \mathrm{~T}$, $P=0$ kbar, $L=8 \mathrm{~nm}$.

Figure 4 The OR coefficient $\chi_{0}^{(2)}$ versus the incident photon energy with three different strength of the magnetic field $B(\mathrm{a})$, and the low-lying energy levels of $E_{0}, E_{1}$, the difference $E_{10}(\mathrm{~b})$ versus the applied magnetic field for $P=0 \mathrm{kbar}, T=0 \mathrm{~K}, L=8 \mathrm{~nm}$.

Figure 5 The SHG susceptibility $\chi_{2 \omega}^{(2)}$ versus the incident photon energy with three different strength of the hydrostatic pressure $P(a)$, and the lowlying energy levels of $E_{0}, E_{1}$, the difference $E_{10}(\mathrm{~b})$ versus the applied magnetic field for $B=0 \mathrm{~T}, T=0 \mathrm{~K}, L=8 \mathrm{~nm}$.

Figure 6 The SHG susceptibility $\chi_{2 \omega}^{(2)}$ versus the incident photon energy with three different strength of the temperature $T(\mathrm{a})$, and the low-lying energy levels of $E_{0}, E_{1}$, the difference $E_{10}(\mathrm{~b})$ versus the applied magnetic field for $B=0 \mathrm{~T}, P=0 \mathrm{kbar}, L=8 \mathrm{~nm}$.

Figure 7 The SHG susceptibility $\chi_{2 \omega}^{(2)}$ versus the incident photon energy with three different strength of the magnetic field $B(\mathrm{a})$, and the low-lying energy levels of $E_{0}, E_{1}$, the difference $E_{10}(\mathrm{~b})$ versus the applied magnetic field for $P=0 \mathrm{kbar}, T=0 \mathrm{~K}, L=8 \mathrm{~nm}$. 
Fig.1

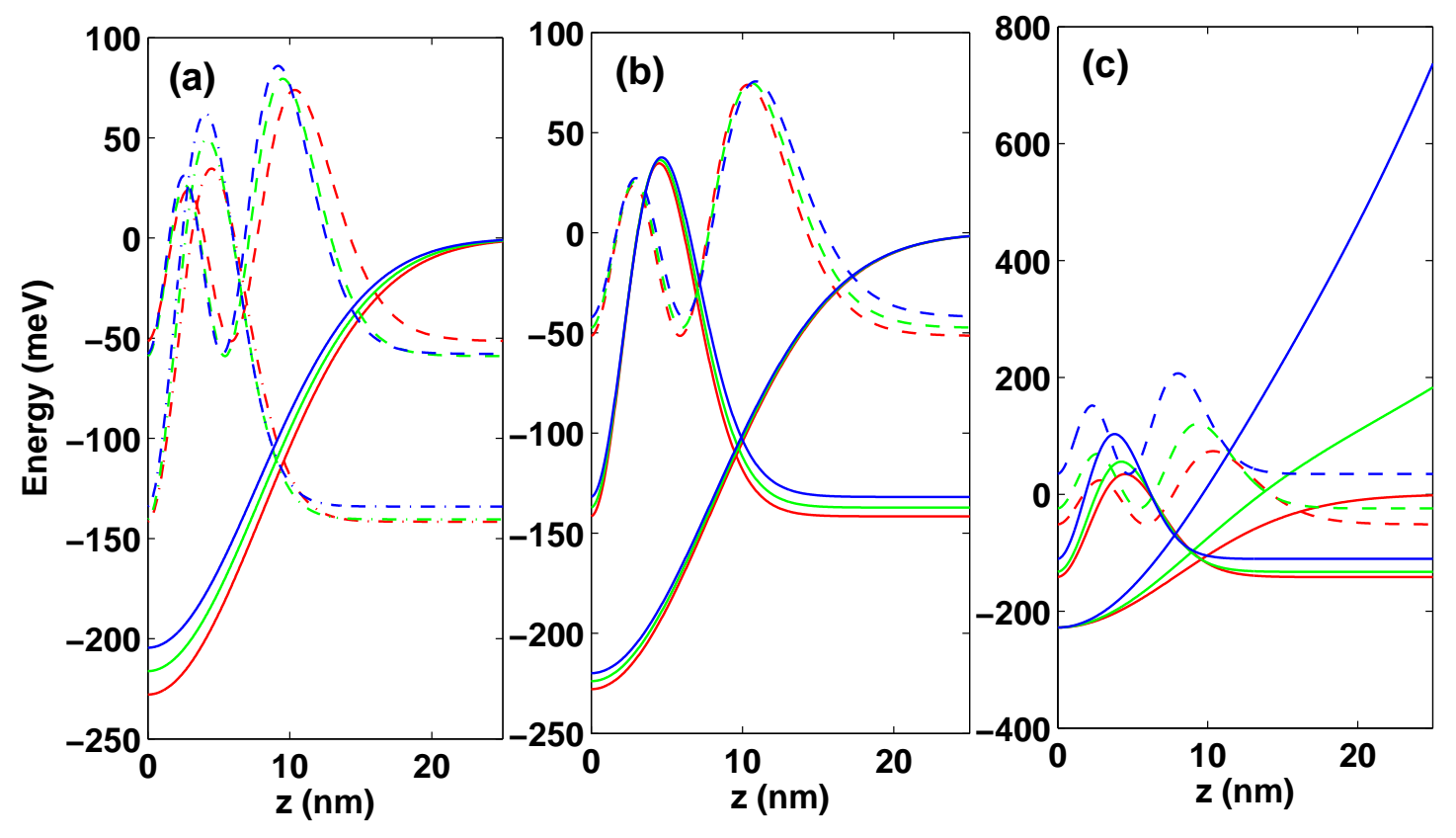




\section{Fig.2}

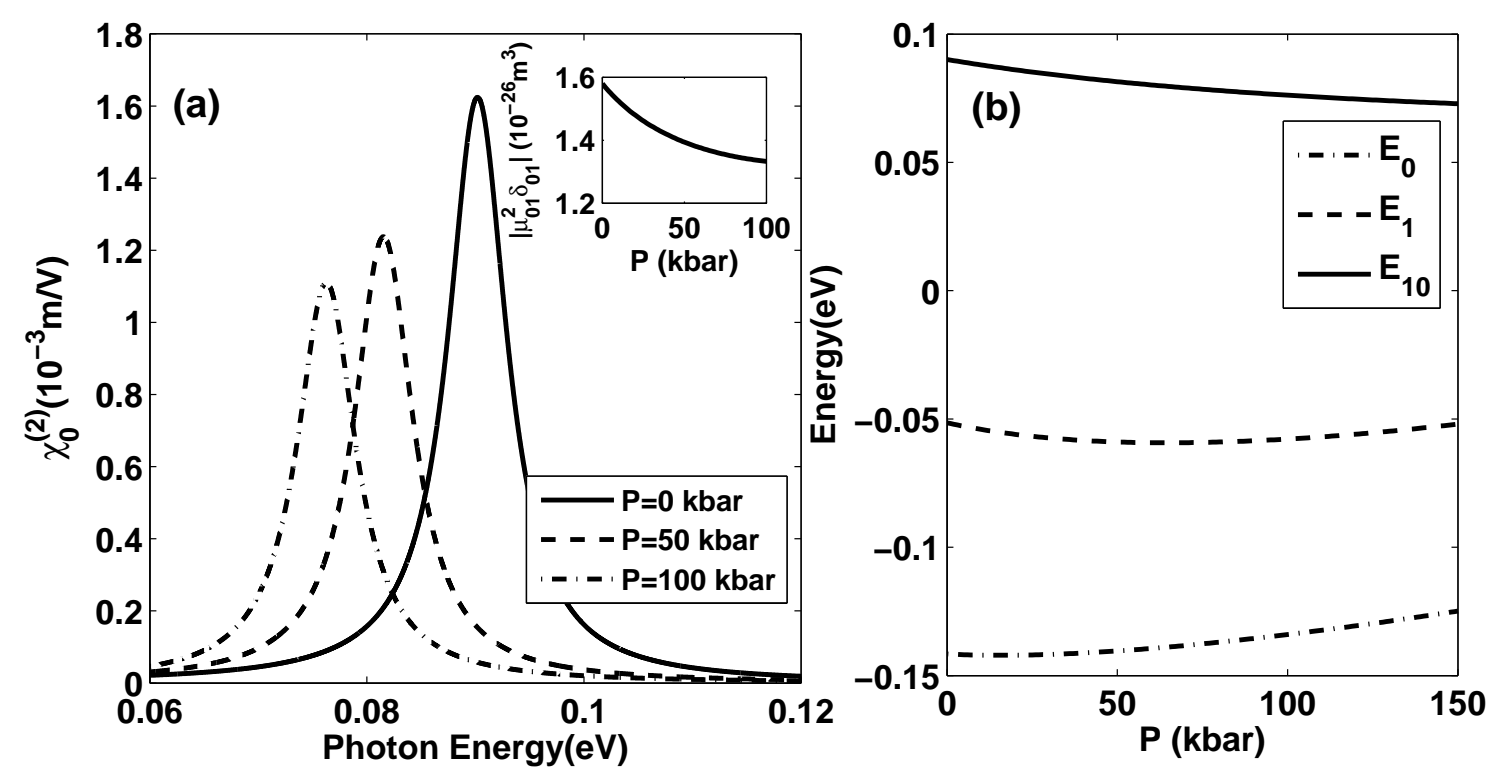


Fig.3

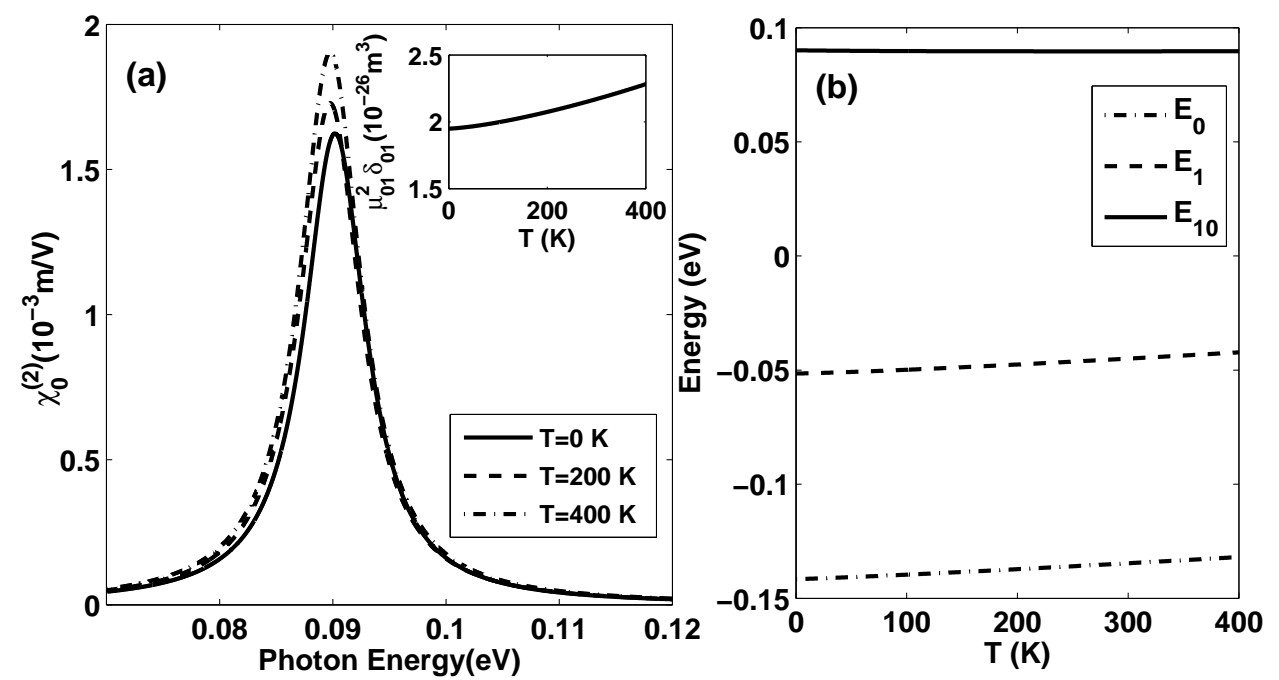


Fig.4
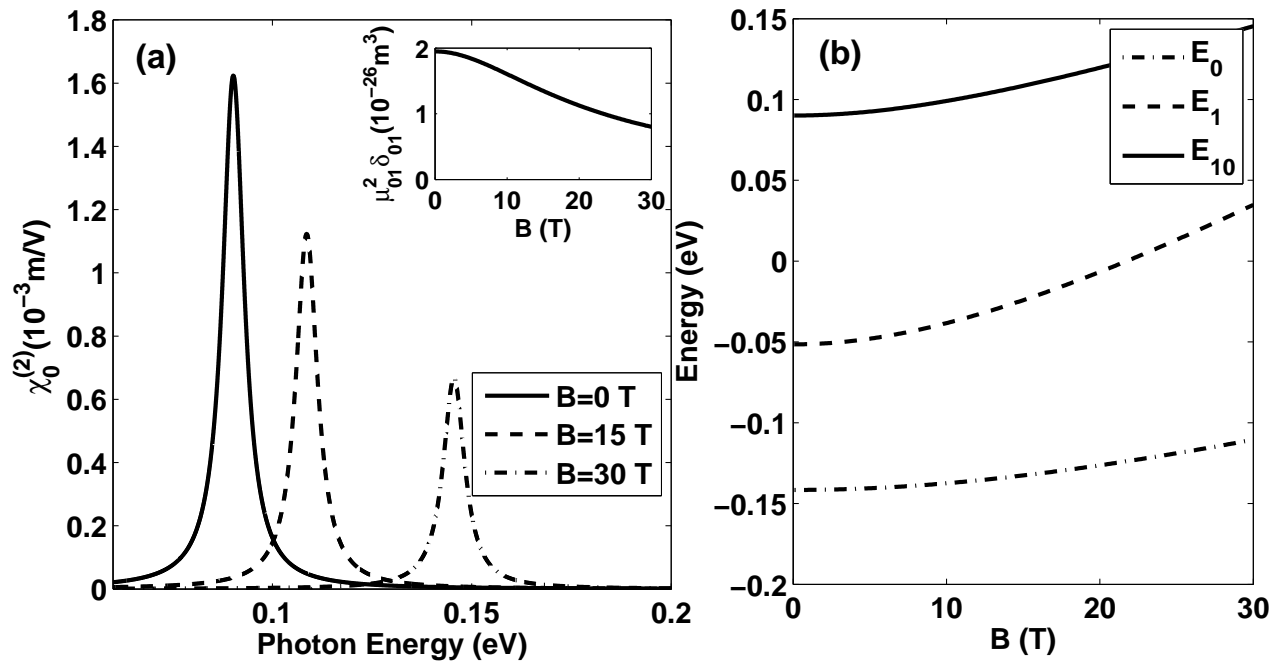
Fig.5
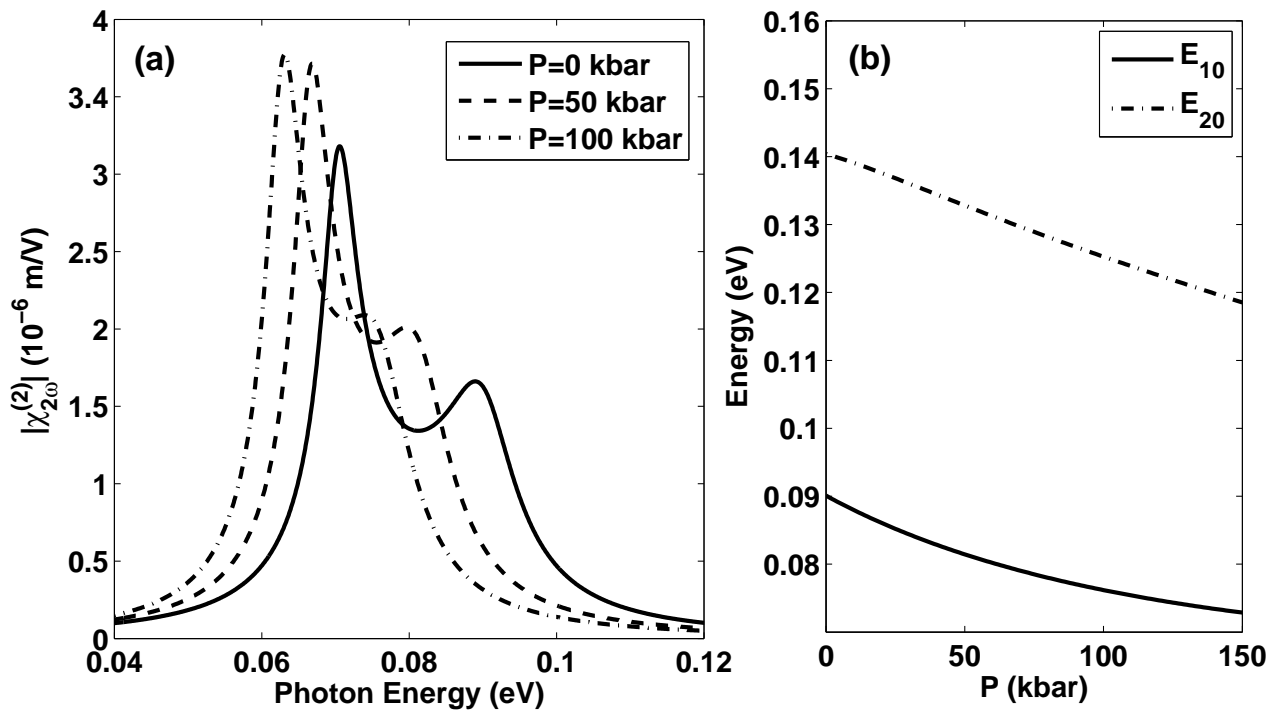
Fig.6
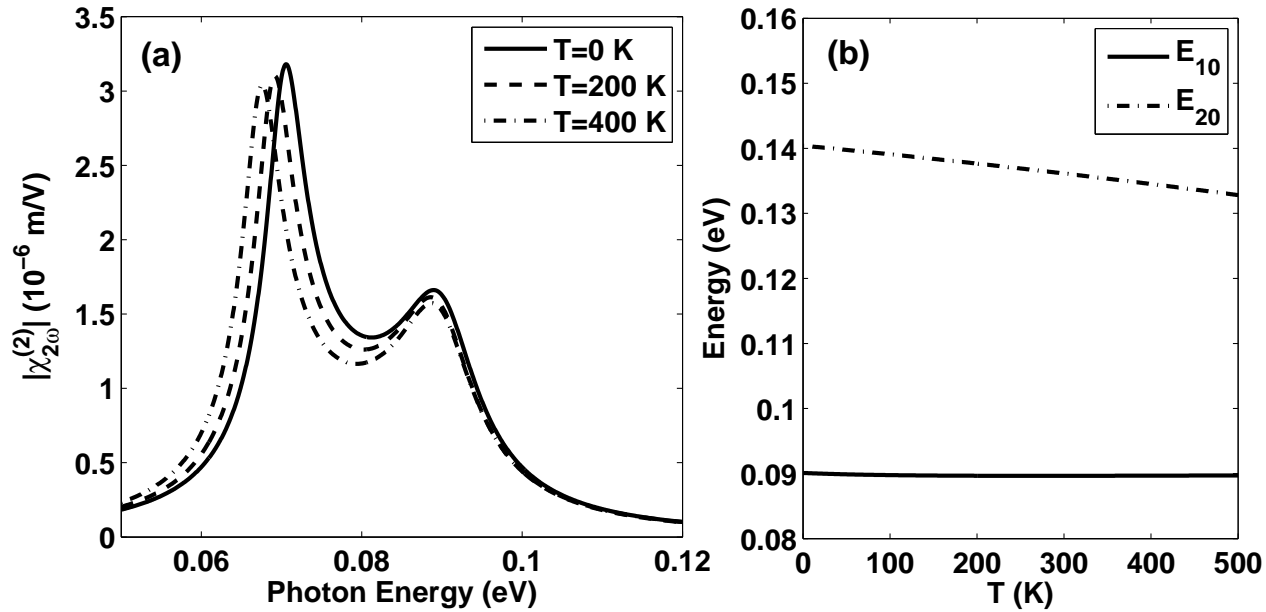
Fig.7
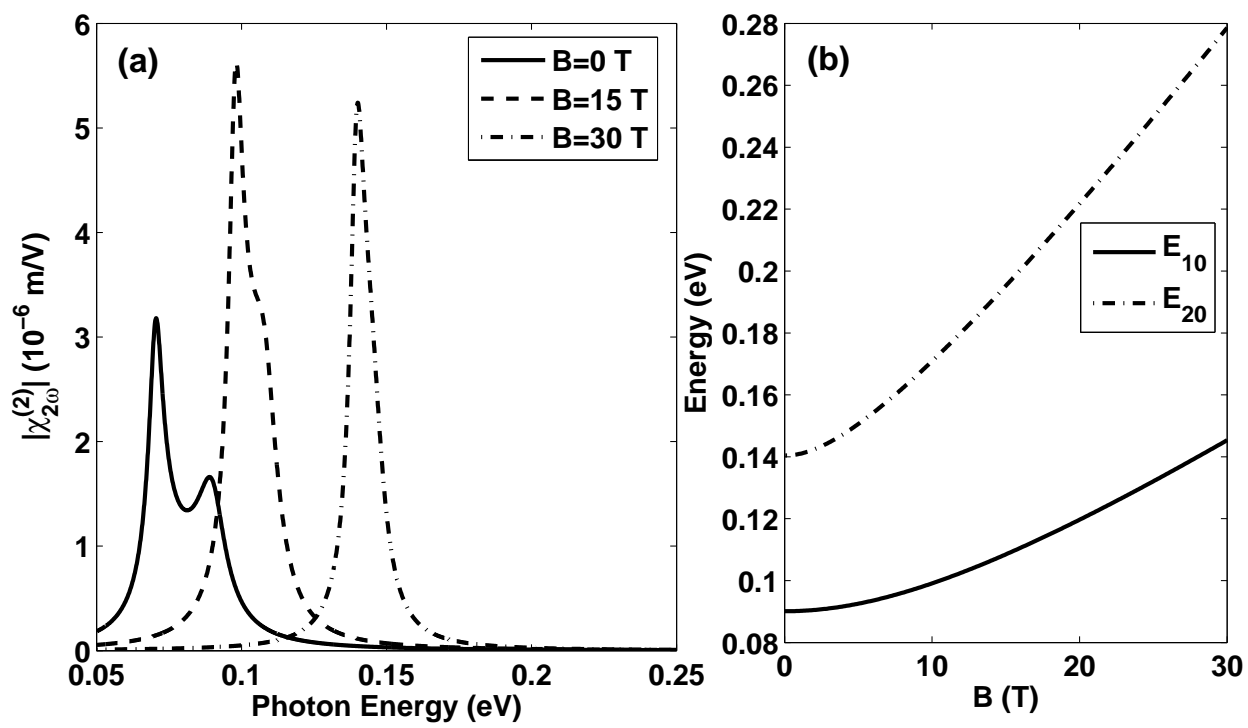